\title{
Establishment of in vivo fluorescence imaging in mouse models of malignant mesothelioma
}

\author{
NORIE YAMAOKA ${ }^{1}$, YOSHIKO KAWASAKI ${ }^{1}$, YUNFENG XU ${ }^{1}$, HIDEYUKI YAMAMOTO ${ }^{1}$, \\ NOBUYUKI TERADA ${ }^{2}$, HARUKI OKAMURA ${ }^{1}$ and SHUJI KUBO ${ }^{1}$ \\ ${ }^{1}$ Laboratory of Host Defenses, Institute for Advanced Medical Sciences, and ${ }^{2}$ Department of Pathology, \\ Hyogo College of Medicine, 1-1 Mukogawa-cho, Nishinomiya, Hyogo 663-8501, Japan \\ Received April 9, 2010; Accepted May 17, 2010
}

DOI: 10.3892/ijo_00000675

\begin{abstract}
Malignant mesothelioma is a highly aggressive tumor with poor prognosis, and new treatment paradigms are urgently needed. For testing preclinical efficacy of new therapeutic agents, establishment of appropriate animal models is crucial. We developed in vivo fluorescence imaging models for human malignant mesothelioma in mice using tumor cells engineered to express fluorescent proteins (EGFP, mRFP, mCherry, and mPlum) by lentiviral vectors. Among these fluorescent proteins, the expression of mCherry protein in the transduced tumor cells was shown to be robust and stable both in vitro and in vivo. In both, peritoneally disseminated and orthotopic pleural mesothelioma models, mCherry-positive tumors were sensitively detected and tumor growth was successfully monitored. This represents the first study to achieve sensitive tumor detection and tracking of tumor growth and development in the malignant mesothelioma mouse models by non-invasive in vivo fluorescence imaging. These imaging models can be versatile and powerful tools to explore new treatment paradigms for malignant mesothelioma.
\end{abstract}

\section{Introduction}

Malignant mesothelioma is a neoplasm of the mesothelial cells lining the pleural or peritoneal cavity, and is linked to prior exposure to asbestos $(1,2)$. It is a highly aggressive tumor with poor prognosis: a median survival duration of $<1$ year and a 5 -year survival rate of $\leq 1 \%$ (2). Conventional therapies for malignant mesothelioma are generally non-curative, and new treatment paradigms are greatly needed. In order to develop new therapeutics and diagnostics, establishment of

Correspondence to: Dr Shuji Kubo, Laboratory of Host Defenses, Institute for Advanced Medical Sciences, Hyogo College of Medicine, 1-1 Mukogawa-cho, Nishinomiya, Hyogo 663-8501, Japan

E-mail: s-kubo@hyo-med.ac.jp

Key words: in vivo fluorescence imaging, malignant mesothelioma 'patient-like' animal tumor models are critical, which can aid in the reliable detection of tumor growth and effective response to therapy (3).

In vivo animal imaging can be a powerful tool in the development and evaluation of diagnostic and therapeutic procedures, in the accurate replication of human cancers, and in enabling in vivo molecular studies. A number of different types of mesothelioma imaging models have been reported, using positron-emission tomography (PET) (4-7), bioluminescence imaging $(8,9)$, and fluorescence imaging $(5,10)$. Fluorescence imaging of cancers in animal models offers many potential advantages, including rapid imaging, high sensitivity, and high time resolution. It is also less expensive than other imaging tools, since visualization of fluorescence images requires no preparative procedures or contrast agents $(11,12)$, and the procedure is less stressful to mice. These advantages make the cells and animal models expressing fluorescent protein(s) very attractive for the evaluation of therapeutics, and especially for high throughput screening.

For ideal in vivo fluorescence imaging, fluorescent proteins with long wavelength (e.g., red) would be preferable because of their superior tissue penetration compared to spectral variants of shorter wavelength. A number of fluorescent proteins have been developed to date. Red-shifted fluorescent proteins, including mCherry [maximum excitation (Ex)/ maximum emission $(\mathrm{Em})=587 / 610 \mathrm{~nm}](13)$ and mPlum $(\mathrm{Ex} / \mathrm{Em}=590 / 649 \mathrm{~nm})(14)$, are mutants derived from monomeric red fluorescent protein $(\mathrm{mRFP} ; \mathrm{Ex} / \mathrm{Em}=584 / 607 \mathrm{~nm})$, a mutant of DsRed from the Discosoma coral, by directed mutagenesis (15). They possess enhanced optical qualities at levels comparable to green fluorescent protein (GFP)-based systems, including enhanced GFP (EGFP: Ex/Em = 488/ $507 \mathrm{~nm})(13)$.

In this study, to visualize tumor cells by using in vivo imaging, we have developed stable fluorescent proteinexpressing mesothelioma cells, including EGFP, mRFP, mCherry, and mPlum. Among these fluorescence-transduced tumor cells, mCherry expression was shown to be robust and stable both in vitro and in vivo. In both, a peritoneally disseminated mesothelioma model and an orthotopic pleural mesothelioma model, tumor nodules were sensitively detected, even in the deep areas of the chest and the abdominal cavity. This represents the first study to show tumor growth in the mesothelioma models by using in vivo fluorescence 
imaging. Our in vivo mCherry fluorescence imaging mesothelioma models are sensitive, feasible, and powerful tools to evaluate their characteristics and usefulness in monitoring tumor growth, possibly in evaluating therapy response and hence, in exploring new treatment paradigms for malignant mesothelioma.

\section{Materials and methods}

Cell lines. The human malignant pleural mesothelioma MSTO-211H cells were obtained from American Type Culture Collection (ATCC, Manassas, VA, USA), and grown in RPMI-1640 (Nacalai Tesque, Kyoto, Japan) supplemented with $10 \%$ heat-inactivated fetal calf serum (FCS; HyClone, Logan, UT, USA). Cells were cultured in humidified $10 \%$ $\mathrm{CO}_{2}$ at $37^{\circ} \mathrm{C}$.

Construction and production of fluorescent protein-expressing lentiviral vectors. The mRFP-, mPlum-, and mCherryfluorescence expression vectors were kindly provided by Roger Y. Tsien (University of California, San Diego). Selfinactivating lentivirus vectors expressing fluorescent proteins, LVSKc-mRFP, LVSKc-mPlum, and LVSKc-mCherry were created from sinSKcmv-EGFP (LVSKc-EGFP) (16), by replacement of EGFP with $\mathrm{mRFP}$, mPlum, and mCherry cDNA, respectively. The virus preparations were produced by transient cotransfection of $293 \mathrm{~T}$ cells as described previously $(16,17)$. The titers of these vectors were determined by fluorescent protein expression using an FACSCalibur flow cytometer (Becton-Dickinson Japan, Tokyo, Japan), and expressed in terms of transducing units (TUs) per milliliter.

Transduction of MSTO-211H mesothelioma cells with fluorescent proteins. MSTO-211H cells were cultured in growth medium to $30-50 \%$ confluency at the time of transduction $\left(1 \times 10^{6}\right.$ cells per well in 6-well plates). Then, the cells were incubated with either LVSKc-mRFP, LVSKc-mPlum, LVSKc-mCherry or LVSKc-EGFP at a multiplicity of infection of 10 for $72 \mathrm{~h}$. Then, the cells were passaged at a ratio of 1:5. High fluorescence expression cell clones were selected in 96-well plates, generating MSTO-mRFP, MSTOmPlum, MSTO-mCherry, and MSTO-EGFP cells. The high fluorescence expression clones were amplified and transferred by conventional culture methods.

In vitro growth curves. MSTO-211H and MSTO-mCherry cells were seeded in triplicate at $5 \times 10^{3}$ per well in 12 -well culture plates. Cells in triplicate wells were harvested daily by trypsinization and the number of viable cells was determined by the trypan blue exclusion assay (Sigma-Aldrich Japan, Tokyo, Japan).

Nude mice. All experiments with mice were conducted according to institutional guidelines under an approved protocol by the Animal Care Committee of Hyogo College of Medicine, and the mice were given utmost human care. Six to 7-week-old female Balb/c nu/nu mice weighing 19-23 g were used. The animals were kept in wire cages in laminar air flow cabinets at a temperature of $21-25^{\circ} \mathrm{C}$, a humidity of $40-60 \%$, and a 12/12-h light/dark cycle.
Subcutaneous tumor growth. Fluorescence-expressing cell lines, MSTO-mRFP, MSTO-mPlum, MSTO-mCherry, and MSTO-EGFP, were harvested at near confluence with $0.05 \%$ trypsin/EDTA solution. One million cells in $100 \mu \mathrm{l}$ of $\mathrm{Ca}^{2+}$ and $\mathrm{Mg}^{2+}$-free Hank's balanced salt solution (HBSS) were injected subcutaneously on the dorsal flank of three nude mice as shown in Fig. 1A. Tumors were analyzed weekly by in vivo imaging. At 3-weeks after injection, tumors were excised, disaggregated with $0.1 \%$ collagenase type IV (Sigma-Aldrich) and $0.01 \%$ Hyaluronidase type V (SigmaAldrich) in HBSS, and replated. The next day, tumor cells were analyzed for fluorescence expression using fluorescence microscope and flow cytometry.

To determine the in vivo growth potential, either $1 \times 10^{6}$ MSTO-211H or MSTO-mCherry cells were injected into the flanks of nude mice ( $n=3$ per cell type). Tumor growth was monitored every week over 13 weeks using a caliper. Tumor volume was calculated as $\alpha \mathrm{x} \mathrm{b}^{2} \times 0.5$ ( $\alpha$, largest diameter; b, smallest diameter).

Intraperitoneal implantation of MSTO-mCherry cells. MSTOmCherry tumor cells $\left(1 \times 10^{6}\right)$ in $0.4 \mathrm{ml}$ HBSS were injected intraperitoneally into nude mice $(\mathrm{n}=3)$. In vivo fluorescence imaging analysis was performed weekly. At 3 weeks after injection, mice were sacrificed and the peritoneal tumors were immediately excised and measured to investigate the relationship between fluorescence signals and actual tumor volumes.

Intrapleural orthotopic implantation of MSTO-211H-mCherry cells. Mice were anaesthetized with sodium pentobarbital (Nembutal) and placed in left lateral decubitus position. A 24-gauge shielded intravenous catheter (BD Insyte Autoguard) (Becton-Dickinson) was advanced approximately through the fourth intercostal space to about $5 \mathrm{~mm}$, into the right pleural cavity, and then MSTO-mCherry tumor cells $\left(5 \times 10^{5}\right.$ in $0.2 \mathrm{ml}$ HBSS/mouse) were injected in the peritoneal cavity of nude mice $(\mathrm{n}=3)$. In vivo fluorescence imaging analysis was performed weekly.

In vivo fluorescence imaging analysis. For in vivo imaging analysis, mice were anesthetized with sodium pentobarbital, and spectral fluorescence imaging was performed using the Maestro in vivo fluorescence imaging system (Cambridge Research and Instrumentation, Woburn, MA, USA), as previously described (18). For detection of four different fluorescence labels, whole body images were captured at a $500-600 \mathrm{~nm}$ range in $10 \mathrm{~nm}$ steps with a set of band-pass filters $(\mathrm{Ex} / \mathrm{Em}=445-490 / 515 \mathrm{~nm})$. For detection of mCherry, images were captured at a 550-800 nm range in $10 \mathrm{~nm}$ steps with a set of band-pass filters $(E x / E m=503-555 / 580 \mathrm{~nm})$. A green filter set of band-pass filters from 503 to $555 \mathrm{~nm}$ and a long-pass filter of $580 \mathrm{~nm}$ were used for excitation and emission light, respectively. All images were analyzed by Maestro 2.2 software in order to create unmixed images of fluorescein and autofluorescence.

Statistical analysis. The results are presented as mean \pm standard deviation (SD). Statistical significance of differences was calculated using Student's t-test, and a $\mathrm{P}<0.01$ was considered significant. 
A

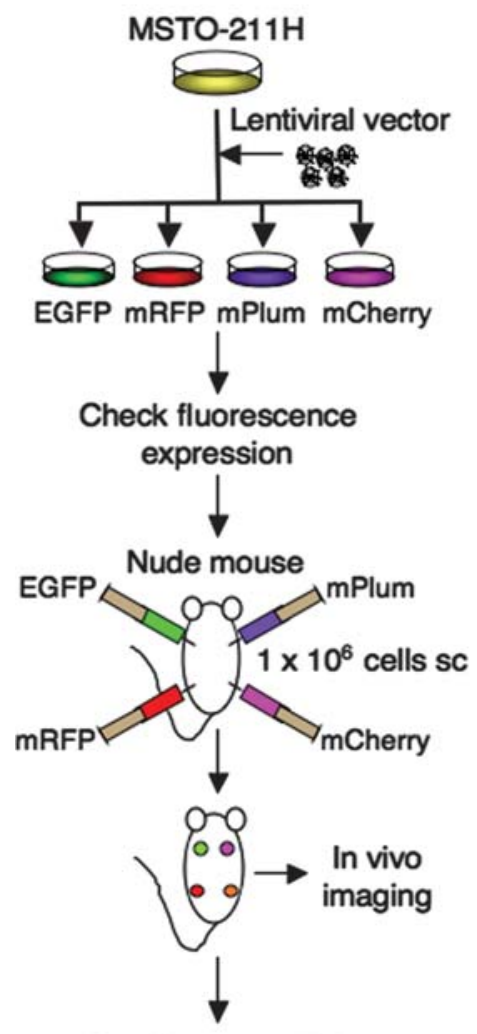

Sacrifice at week 3

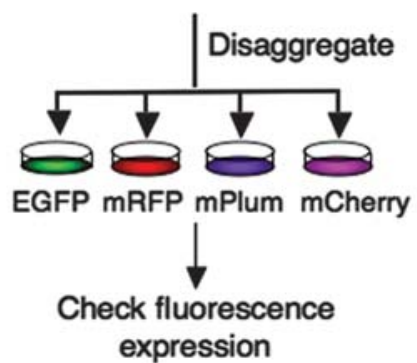

B
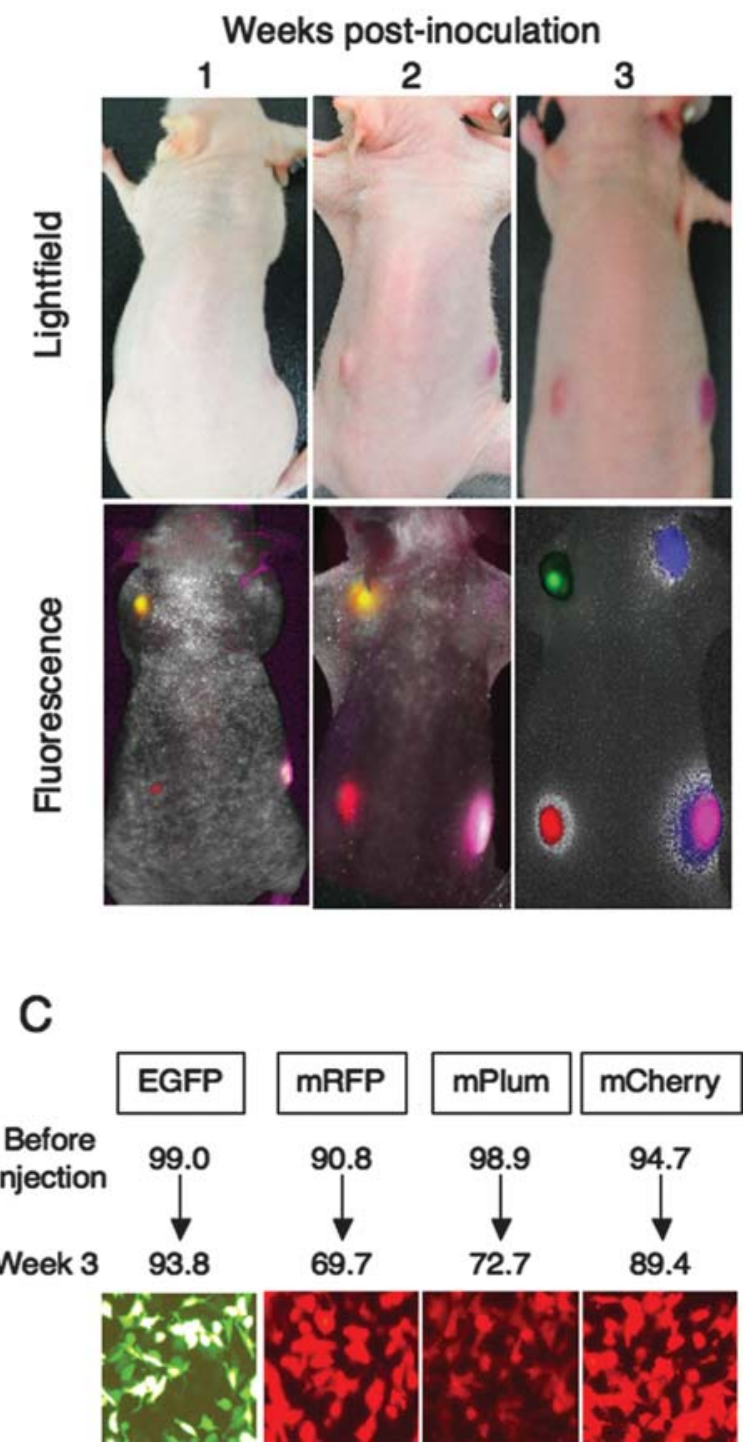

Figure 1. Establishment of fluorescence-labeled human malignant mesothelioma cell lines and stability of fluorescence expression after in vivo passage in nude mice. Human malignant mesothelioma MSTO-211H cell line was transduced by lentivirus, resulting in high-level expression of EGFP, mRFP, mPlum, and mCherry fluorescent proteins (A, see also Materials and methods). Each cell clone was first analyzed for fluorescence expression using flow cytometer and then $1 \times 10^{6}$ cells were subcutaneously (sc) injected into different sites on the flank of the same nude mice as shown in the figure. Tumors were analyzed by in vivo imaging weekly up to 3 weeks post-inoculation (B, representative images from three mice are shown). At 3 weeks after injection, tumors were excised, disaggregated, and replated. The next day, tumor cells were analyzed for fluorescence expression using fluorescence microscopy and flow cytometry. The transduction efficiencies before injection and at 3 weeks after injection are depicted (C).

\section{Results}

Transduction and selection of high mCherry-expression MSTO-211H mesothelioma cells. In order to obtain fluorescent cells, human malignant mesothelioma MSTO-211H cell line was transduced by each lentivirus vector, and selected to generate stable cell lines expressing high levels of fluorescent proteins; MSTO-EGFP, MSTO-mRFP, MSTO-mPlum, and MSTO-mCherry cells. To examine which cells are suitable for in vivo imaging, $1 \times 10^{6}$ cells of each cell clone were subcutaneously injected into different sites of the flank of the same nude mouse (Fig. 1A). Tumor formation was observed in all the different cell clones in all mice, and no significant difference in tumor size was found among the cell clones. Among these cells, MSTO-mCherry showed robust fluorescence expression
(Fig. 1B), with peak fluorescence intensity at 3 weeks postinoculation more than 3-fold higher than MSTO-EGFP, MSTO-mRFP, or MSTO-mPlum. Additionally, mCherry protein was stably expressed in MSTO-mCherry cells after disaggregation of the subcutaneous tumors (Fig. 1C). For these reasons, we have chosen to use MSTO-mCherry cells for further in vivo imaging work.

Biological properties of mCherry-expressing MSTO-211H cells. We next investigated biological properties of MSTOmCherry cells since fluorescent proteins are potentially toxic for cells (13). The mCherry expression remained stable at least for 3 months in MSTO-mCherry cells, and was easily monitored by fluorescence microscopy (Fig. 2A). Flow cytometric analysis at 3 months demonstrated that MSTO-mCherry 
A MSTO-211H
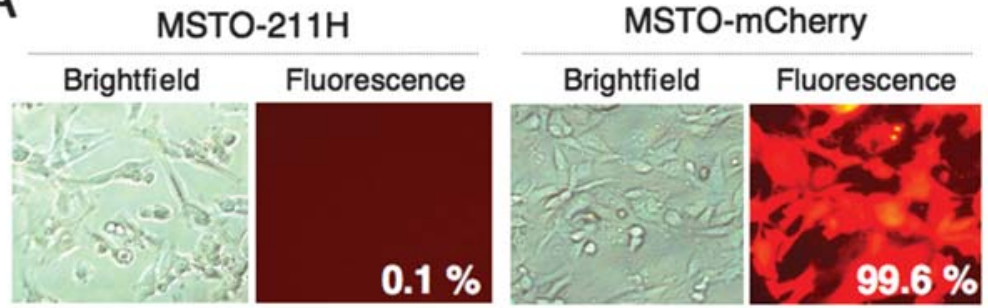

B
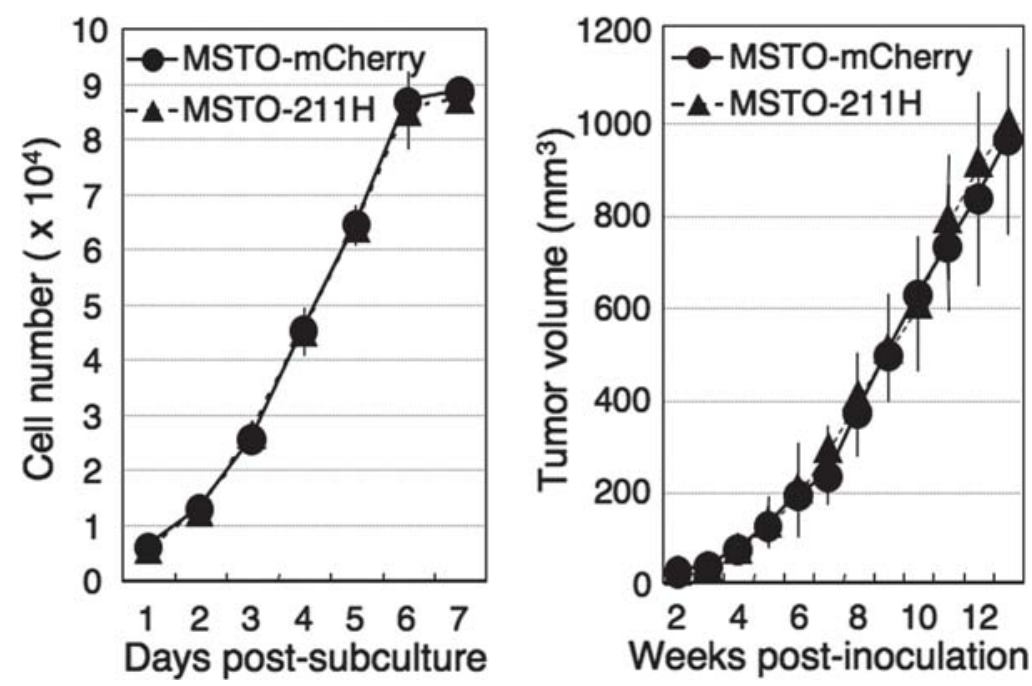

Figure 2. Biological properties of mCherry-expressing MSTO-211H cells. Biological properties of MSTO-mCherry cells were compared with non-transduced parent MSTO-211H cells. (A) The mCherry fluorescence expression under microscopy. The percentage of mCherry-positive cells after 3-months culture is depicted in the figure. (B) In vitro growth of MSTO-211H and MSTO-mCherry cells. (C) In vivo growth of MSTO-211H and MSTO-mCherry cells .

cells were $99.6 \%$ mCherry-positive, suggesting stable expression via a stable chromosomal integration of the mCherryexpression gene cassette by the lentiviral vector.

To determine the possible alteration of any biological properties in MSTO-mCherry cells, these cells were compared with non-transfected parental MSTO-211H cells. First, there was no difference in cell morphology (Fig. 2A). Also, no difference was observed in the in vitro growth rate between MSTO-211H and MSTO-mCherry cells by 7 days as determined by comparison of their proliferation in monolayer cultures (Fig. 2B).

To compare in vivo tumor formation and growth of MSTO$211 \mathrm{H}$ and MSTO-mCherry cells, nude mice received a subcutaneous injection of MSTO-211H and MSTO-mCherry cells ( $n=3$ per cell type). All of the mice had subcutaneous tumors and there was no difference in tumor size between tumors produced by the MSTO-211H and MSTO-mCherry $>3$ months (Fig. 2C). The subcutaneous fluorescent tumor also showed parental morphology as assessed by histopathological analysis of sections stained with hematoxylin $(\mathrm{H})$ and eosin $(\mathrm{E})$ (data not shown). These results showed that the expression of mCherry fluorescence protein in MSTO-mCherry cells does not affect the tumorigenicity and tumor growth in vivo.

Whole-body fluorescence imaging of intraperitoneal mesothelioma xenotransplant model. We first created an intra- peritoneal mesothelioma xenotransplant model. Following an intraperitoneal injection of MSTO-mCherry cells into nude mice, mCherry-positive tumors became visible by noninvasive in vivo fluorescence imaging in the live animals at one week after inoculation of the tumor cells, and the signal intensities increased over time (Fig. 3A). Intraperitoneally transplanted MSTO-mCherry cells produced extensive disseminated metastasis. At the time of autopsy, all mice had ascites and extensively widespread metastatic foci in the greater omentum, intestinal and mesenteric lymph nodes, and retroperitoneum (Fig. 3B). Fluorescence imaging enabled the identification of extremely small micrometastases $(<1 \mathrm{~mm})$. To investigate the relationship between fluorescence signals and actual tumor volumes, the peritoneal tumors were immediately excised and measured at 3-weeks post-inoculation, and the signal intensities (tumor area) were plotted against tumor volumes. The signal intensities significantly correlated with the MSTO-mCherry tumor volumes $\left(\mathrm{r}^{2}=0.9139 ; \mathrm{P}=0.0029\right)$ (Fig. 3C). Solid tumors were found to have histological features consistent with mesothelioma upon examination of H\&Estained tissue sections, but no evidence of extra-abdominal metastases was shown histologically in any mouse (data not shown).

Whole-body fluorescence imaging in an orthotopic xenotransplant mesothelioma model. We next created an orthotopic 
A

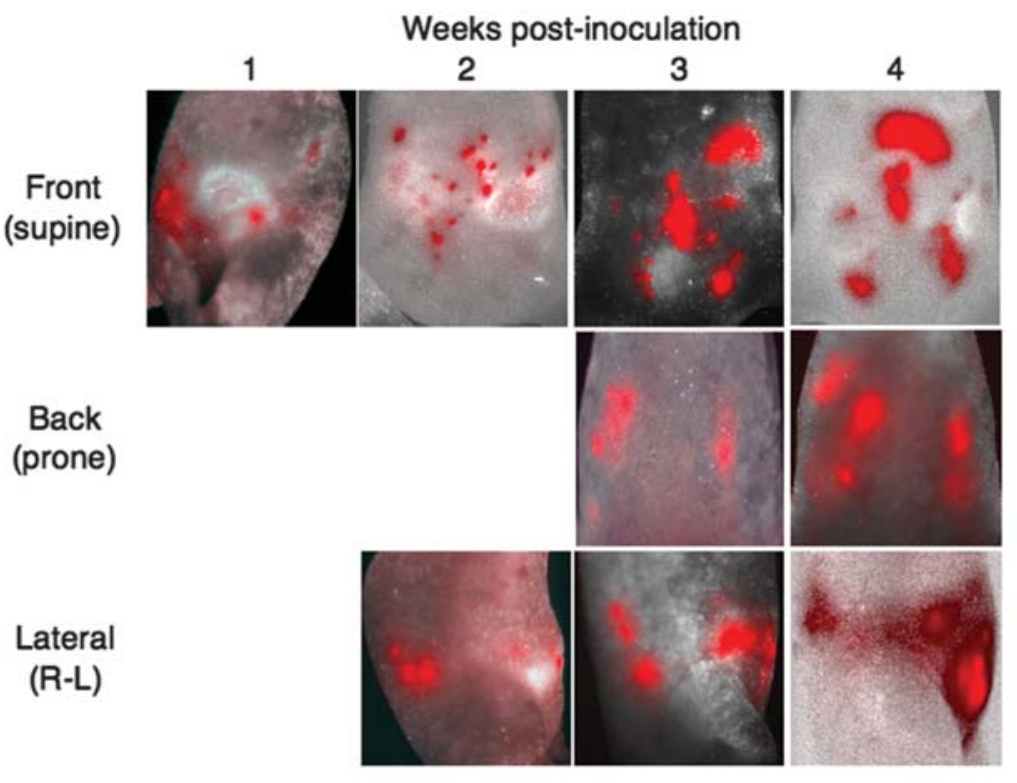

B

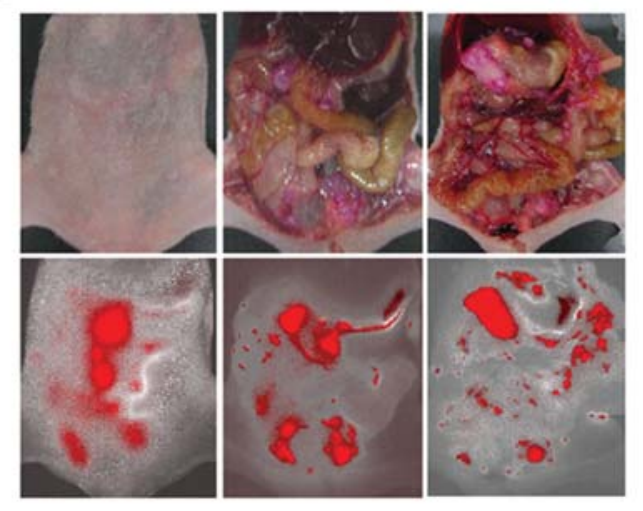

C

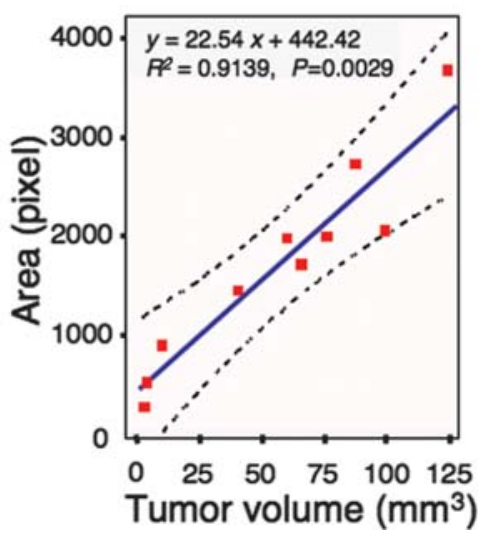

Figure 3. Whole-body fluorescence imaging in an intraperitoneal mesothelioma xenotransplant model. (A) In vivo fluorescence imaging of intraperitoneal (i.p.) tumors. Nude mice were i.p. injected with $1 \times 10^{6}$ MSTO-mCherry cells. Whole-body images were taken weekly under fluorescence light after tumor cell injection. Representative images from three mice are shown. (B) Postmortem fluorescence imaging. Representative images of the same mouse as in A are shown. (C) Relationship between fluorescence signals and actual tumor volumes. After imaging at 3 weeks, the peritoneal tumors were excised and measured, and the signal intensities (area/pixels) were plotted against tumor volumes. Solid line, linear regression curve; dashed lines, $95 \%$ confidence interval $\left(r^{2}=0.91\right.$; $\mathrm{P}<0.0029$ ).

xenotransplant mesothelioma model. Following an intrathoracic injection of MSTO-mCherry cells into nude mice, MSTO-mCherry tumors became visible non-invasively by in vivo fluorescence imaging in the live animals within one week after tumor implantation, and the signal intensities increased over time (Fig. 4A). Interestingly, by one week after implantation of MSTO-mCherry tumor cells in right pleural cavity, spread of the tumor was observed from the right to the left pleural cavity. At the time of autopsy, fluorescence imaging enabled the identification of disseminated micrometastases in both, pleural cavities and pericardial spaces (Fig. 4B), as confirmed by histological examination. Tumors in the pleural cavity were detected even when located just behind the ribs (T1: Fig. 4B and C, left) and the sternal bone (Fig. 4B). Lung invasion was found in one of the mice (T2: Fig. 4B and C, right). However, no distant metastasis to major organs (liver, spleen, kidneys, intestine, brain) was detected in the mice, which was also confirmed by H\&E staining.

\section{Discussion}

The increasing global incidence of malignant mesothelioma is expected to result in the death of hundreds of thousands of people; this provides a strong moral and social imperative for urgent and focused research (1). In order to develop new therapeutics and diagnostics for this malignancy, ideal animal tumor models are entreated, which can aid in the reliable detection of tumor growth and effective response to therapy (3). In this study, to visualize tumor cells by using non-invasive in vivo imaging, we have compared the feasibility of labeling MSTO-211H human mesothelioma cells stably, with four different fluorescent proteins (EGFP, mRFP, mCherry, and mPlum) using lentiviral vectors. Among these fluorescencetransduced tumor cells, mCherry expression was shown to be robust and stable both in vitro and in vivo. Additionally, mCherry expression did not alter any biological properties of MSTO-mCherry cells in terms of tumorigenicity and tumor 
A
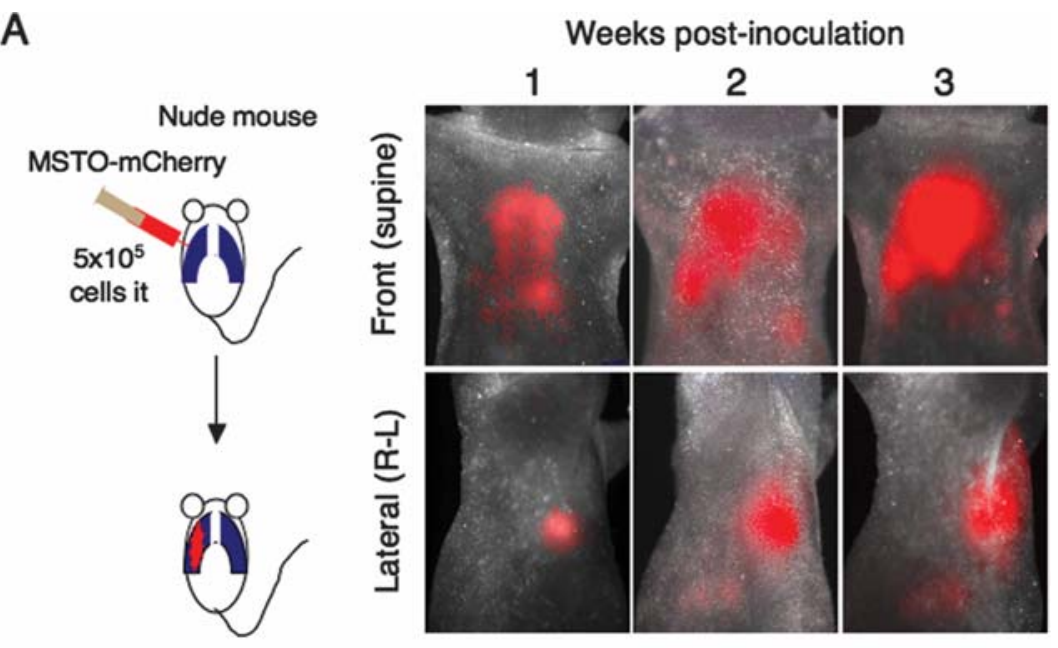

B
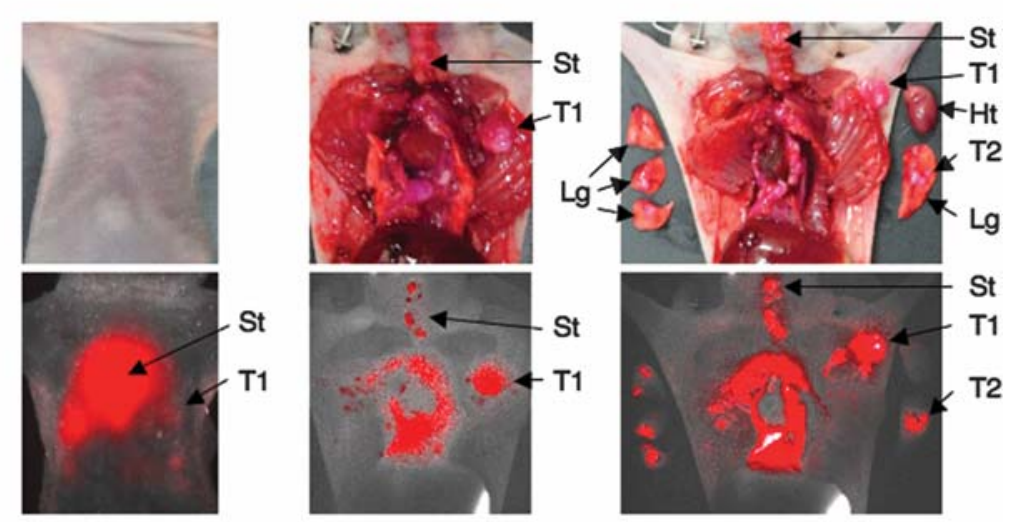

C
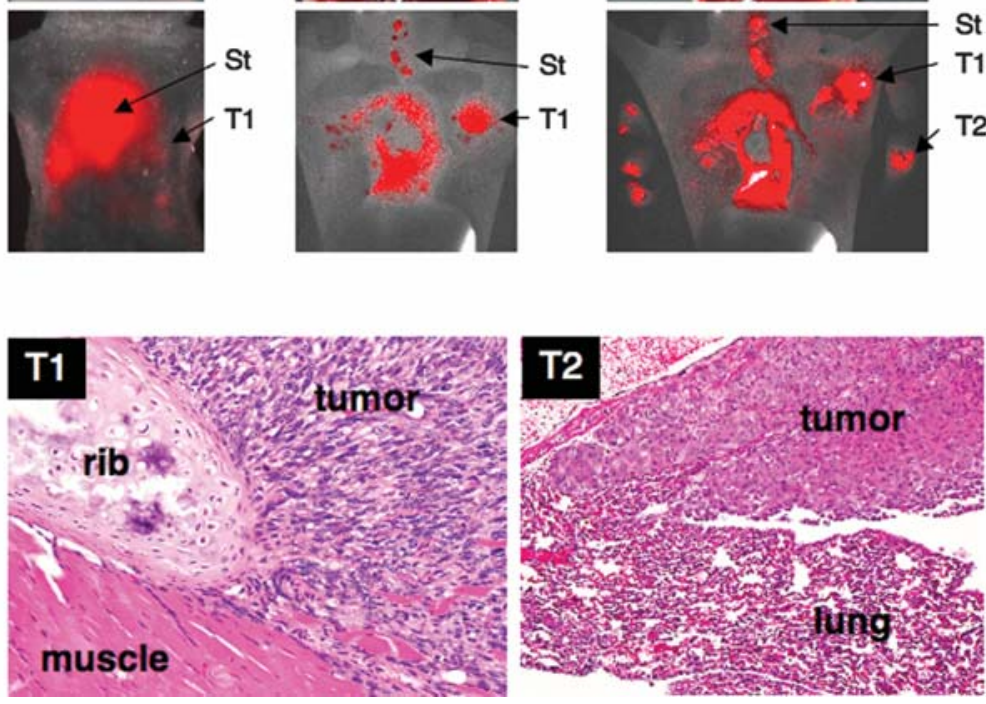

Figure 4. Whole-body fluorescence imaging in an orthotopic mesothelioma model. (A) In vivo fluorescence imaging of pleural tumors. Nude mice were intrathoracically (i.t.) injected with $5 \times 10^{5}$ MSTO-mCherry tumor cells and fluorescence images were taken weekly after injection. Representative images from three mice are shown. (B) Postmortem fluorescence imaging. The fluorescence image of pleural tumors expressing MSTO-mCherry was taken in vivo (A, left) or in situ after the mouse was sacrificed. Representative images of the same mouse as in Fig. 3A are shown. St, sternal bone; Lg, lung; Ht, heart; T1, a tumor behind a rib; T2, a tumor that invaded left lung. (C) Histology of intrathoracic MSTO tumor, H\&E (x200).

growth in vivo. This strong and stable expression of the longwavelength mCherry fluorescent proteins enabled us to develop a peritoneally disseminated mesothelioma model and an orthotopic pleural mesothelioma model, in which tumor nodules could be sensitively detected and monitored for their growth and development by non-invasive in vivo imaging.

In both, peritoneally disseminated and orthotopic pleural mesothelioma models, mCherry-positive tumors were sensitively detected, and tumor growth was successfully monitored. No distant metastasis to major organs were detected in these models, and these results agreed with previous experimental animal studies (19-21) and clinical findings $(22,23)$ that malignant mesothelioma grows locally and rarely exhibits early metastasis to distant sites. However, in orthotopic pleural mesothelioma models, MSTO-mCherry tumor cells were observed to spread from the right to the left pleural cavity within one week after implantation of the tumor cells in the right pleural cavity. This finding is not observed in patients with malignant pleural mesothelioma, possibly because the pericardial cavity in rodents intercommunicates with the adjacent pleural cavities through pericardial pores (24), which do not exist in humans.

There have only been two prior studies reporting in vivo fluorescence imaging of malignant mesothelioma in animal models $(5,10)$. In the first study, Adusumilli et al (10) have reported the capability of in vivo fluorescence imaging to 
demonstrate virus spread in a pleural mesothelioma mouse model treated with replication-competent herpes simplex virus bearing GFP. Although they could detect virus-infected tumor cells indirectly by using replicating virus capable of increasing the copy number of the GFP-expression cassette in the infected cells, they needed a thoracoscope fitted with fluorescence filters for GFP to detect low fluorescence signals due to a low tissue penetration of GFP. In the second study, Saito et al (5) have compared PET tracer uptake and fluorescence imaging of RFP-labeled tumor cells in mesothelioma xenograft mouse models. They showed a nice correlation between fluorescence intensity and tumor weight in subcutaneous and pleural tumor models. However, they showed only one set of low resolution in vivo images of the pleural tumor. In contrast, using noninvasive whole-body fluorescence imaging, we achieved sensitive detection of mesothelioma tumors, and also succeeded in monitoring tumor growth and development in both, peritoneal and pleural cavities.

In conclusion, we have developed in vivo mCherry fluorescence imaging models for malignant mesothelioma which facilitate real-time sequential fluorescence imaging to detect tumors and semiquantify tumor burden without invasive procedures in live animals. This tumor model provided us with the opportunity to demonstrate the capability of fluorescent mCherry to detect and visualize dissemination and micrometastases of tumors and thus its versatility in detection and treatment monitoring of malignant mesothelioma.

\section{Acknowledgements}

We thank Kenta Kobayashi and members of the Joint-Use Research Facilities of the Hyogo College of Medicine for their technical assistance; Roger Y. Tsien (University of California, San Diego) for mRFP-, mPlum-, and mCherry-fluorescence expression vectors. This study was supported by Osaka Cancer Research Foundation; a Grant-in-Aid for Promotion of Technical Seeds in Advanced Medicine, Hyogo College of Medicine; a Grant-in-Aid for Scientific Research from the Ministry of Education, Culture, Sports, Science and Technology of Japan.

\section{References}

1. Robinson BW and Lake RA: Advances in malignant mesothelioma. N Engl J Med 353: 1591-1603, 2005.

2. Ceresoli GL, Locati LD, Ferreri AJ, et al: Therapeutic outcome according to histologic subtype in 121 patients with malignant pleural mesothelioma. Lung Cancer 34: 279-287, 2001.

3. Colt HG, Astoul P, Wang X, Yi ES, Boutin C and Hoffman RM: Clinical course of human epithelial-type malignant pleural mesothelioma replicated in an orthotopic-transplant nude mouse model. Anticancer Res 16: 633-639, 1996.

4. Brader P, Kelly KJ, Chen N, et al: Imaging a genetically engineered oncolytic vaccinia virus (GLV-1h99) using a human norepinephrine transporter reporter gene. Clin Cancer Res 15: 3791-3801, 2009.
5. Saito Y, Furukawa T, Arano Y, Fujibayashi Y and Saga T: Comparison of semiquantitative fluorescence imaging and PET tracer uptake in mesothelioma models as a monitoring system for growth and therapeutic effects. Nucl Med Biol 35: 851-860, 2008.

6. Tsuji AB, Sogawa C, Sugyo A, et al: Comparison of conventional and novel PET tracers for imaging mesothelioma in nude mice with subcutaneous and intrapleural xenografts. Nucl Med Biol 36: 379-388, 2009.

7. Zhuang H, Pourdehnad M, Lambright ES, et al: Dual time-point 18F-FDG PET imaging for differentiating malignant from inflammatory processes. J Nucl Med 42: 1412-1417, 2001.

8. Chang CL, Wu TC and Hung CF: Control of human mesothelinexpressing tumors by DNA vaccines. Gene Ther 14: 1189-1198, 2007.

9. Bertino P, Piccardi F, Porta C, et al: Imatinib mesylate enhances therapeutic effects of gemcitabine in human malignant mesothelioma xenografts. Clin Cancer Res 14: 541-548, 2008.

10. Adusumilli PS, Stiles BM, Chan MK, et al: Imaging and therapy of malignant pleural mesothelioma using replication-competent herpes simplex viruses. J Gene Med 8: 603-615, 2006.

11. Blasberg RG and Gelovani J: Molecular-genetic imaging: a nuclear medicine-based perspective. Mol Imaging 1: 280-300, 2002.

12. McVeigh ER: Emerging imaging techniques. Circ Res 98: 879-886, 2006.

13. Shaner NC, Campbell RE, Steinbach PA, Giepmans BN, Palmer AE and Tsien RY: Improved monomeric red, orange and yellow fluorescent proteins derived from Discosoma sp. red fluorescent protein. Nat Biotechnol 22: 1567-1572, 2004.

14. Wang L, Jackson WC, Steinbach PA and Tsien RY: Evolution of new nonantibody proteins via iterative somatic hypermutation. Proc Natl Acad Sci USA 101: 16745-16749, 2004.

15. Campbell RE, Tour O, Palmer AE, et al: A monomeric red fluorescent protein. Proc Natl Acad Sci USA 99: 7877-7882, 2002.

16. Kubo S, Seleme MC, Soifer HS, et al: L1 retrotransposition in nondividing and primary human somatic cells. Proc Natl Acad Sci USA 103: 8036-8041, 2006.

17. Kubo S and Mitani K: A new hybrid system capable of efficient lentiviral vector production and stable gene transfer mediated by a single helper-dependent adenoviral vector. J Virol 77: 2964-2971, 2003.

18. Satake A, Inoue $\mathrm{T}$, Kubo $\mathrm{S}$, et al: Separation of antileukemic effects from graft-versus-host disease in MHC-haploidentical murine bone marrow transplantation: participation of host immune cells. Int J Hematol 91: 485-497, 2010.

19. Craighead JE, Akley NJ, Gould LB and Libbus BL: Characteristics of tumors and tumor cells cultured from experimental asbestos-induced mesotheliomas in rats. Am J Pathol 129: 448-462, 1987.

20. Esandi MC, van Someren GD, Vincent AJ, et al: Gene therapy of experimental malignant mesothelioma using adenovirus vectors encoding the HSV-tk gene. Gene Ther 4: 280-287, 1997.

21. Nakataki E, Yano S, Matsumori Y, et al: Novel orthotopic implantation model of human malignant pleural mesothelioma (EHMES-10 cells) highly expressing vascular endothelial growth factor and its receptor. Cancer Sci 97: 183-191, 2006.

22. Dutt PL, Baxter JW, O'Malley FP, Glick AD and Page DL: Distant cutaneous metastasis of pleural malignant mesothelioma. J Cutan Pathol 19: 490-495, 1992

23. Sterman DH, Recio A, Vachani A, et al: Long-term follow-up of patients with malignant pleural mesothelioma receiving high-dose adenovirus herpes simplex thymidine kinase/ ganciclovir suicide gene therapy. Clin Cancer Res 11: 74447453,2005

24. Nakatani T, Shinohara H, Fukuo Y, Morisawa S and Matsuda T: Pericardium of rodents: pores connect the pericardial and pleural cavities. Anat Rec 220: 132-137, 1988. 